\title{
USO DE PERFIS CROMATOGRÁFICOS DE VOLÁTEIS DE CAFÉS ARÁBICAS TORRADOS PARA A DIFERENCIAÇÃO DAS AMOSTRAS SEGUNDO O SABOR, O AROMA E A QUALIDADE GLOBAL DA BEBIDA
}

\author{
Juliano S. Ribeiro, Fabio Augusto e Márcia M. C. Ferreira* \\ Instituto de Química, Universidade Estadual de Campinas, CP 6154, 13083-970 Campinas - SP, Brasil \\ Terezinha J. G. Salva \\ Centro de Análise e Pesquisa Tecnológica do Agronegócio do Café "Alcides Carvalho", Instituto Agronômico de Campinas, \\ CP 28, 13001-970 Campinas - SP, Brasil
}

Recebido em 4/2/10; aceito em 14/6/10; publicado na web em 21/9/10

\begin{abstract}
THE USE OF CHROMATOGRAPHIC PROFILES FROM ROASTED ARABICA COFFEES TO DIFFERENTIATE SAMPLES ACCORDING TO CLEANLINESS, FLAVOUR AND OVERALL QUALITY OF THE BEVERAGE. In this work, the volatile chromatographic profiles of roasted Arabica coffees, previously analyzed for their sensorial attributes, were explored by principal component analysis. The volatile extraction technique used was the solid phase microextraction. The correlation optimized warping algorithm was used to align the gas chromatographic profiles. Fifty four compounds were found to be related to the sensorial attributes investigated. The volatiles pyrrole, 1-methyl-pyrrole, cyclopentanone, dihydro-2-methyl-3-furanone, furfural, 2-ethyl-5methyl-pyrazine, 2-etenyl-n-methyl-pyrazine, 5-methyl-2-propionyl-furan compounds were important for the differentiation of coffee beverage according to the flavour, cleanliness and overall quality. Two figures of merit, sensitivity and specificity (or selectivity), were used to interpret the sensory attributes studied.
\end{abstract}

Keywords: solid phase microextraction (SPME); coffee flavor; figures of merit.

\section{INTRODUÇÃO}

O aroma do café torrado é constituído por uma mistura complexa de compostos com poderes odoríferos distintos presentes em diferentes concentrações. ${ }^{1}$ De acordo com De Maria et al., ${ }^{2}$ uma mesma substância volátil pode causar uma sensação odorífica agradável ou desagradável, dependendo de sua concentração no meio e das interações com outros compostos.

Atualmente, a qualidade final da bebida do café é determinada pela "prova de xícara", realizada por provadores experientes. Dentre os atributos avaliados por estes profissionais encontram-se a qualidade global, o aroma e o sabor (bebida).

$\mathrm{Na}$ "prova de xícara", considera-se o atributo "aroma" como a percepção olfativa dos gases liberados da infusão de café torrado e moído, enquanto o "sabor ou bebida" é considerado a combinação de sensações causadas pelos compostos químicos da bebida quando introduzida na boca. O atributo "qualidade global", por sua vez, é a percepção conjunta do sabor, do aroma e de outros atributos avaliados durante a análise. ${ }^{3}$ Estas definições acrescidas de resultados disponíveis na literatura indicam a elevada relação entre esses três atributos sensoriais da bebida. ${ }^{4}$

Embora as pesquisas enfatizem que o aroma do café torrado tem um papel fundamental na qualidade da bebida, sendo considerado por muitos como sua impressão digital, são recentes os trabalhos que visam estabelecer essa relação. ${ }^{4-7}$

Além disso, apenas alguns estudos são baseados na análise quimiométrica de dados cromatográficos com o propósito de tratar conjuntamente os constituintes voláteis do café e os resultados da "prova de xícara"., $, 5,8$

Os métodos quimiométricos são especialmente indicados para a análise de cromatogramas de matrizes complexas, envolvendo centenas de compostos, da mesma forma que outros sinais instrumentais. A vantagem em se usar os perfís cromatográficos diretamente, ao invés de suas áreas de picos, é a possibilidade da extração de informações adicionais que não são computadas quando apenas as áreas são utilizadas como resposta analítica. Entretanto, para a aplicação das técnicas quimiométricas, os cromatogramas devem ser adequadamente pré-tratados.

Um aspecto importante a ser considerado quando se analisa diretamente os cromatogramas (como perfis) é o deslocamento de picos que ocorre com frequência, em decorrência de variações na temperatura, idade da coluna, pequenas variações na composição da fase móvel e diferentes maneiras de injeção da amostra. ${ }^{9} \mathrm{O}$ pré-tratamento aplicado a fim de corrigir esses deslocamentos é o alinhamento dos picos dos cromatogramas. Várias técnicas de alinhamento foram propostas na literatura. Dentre elas encontram-se correlation optimized warping (COW) ${ }^{10-12}$ dynamic time warping (DTW), ${ }^{13}$ parametric time warping (PTW), ${ }^{14}$ alinhamento de picos por algoritmos genéticos (PAGA), ${ }^{15}$ fuzzy warping $(\mathrm{FW})^{16}$ e semi-parametric time warping (STW). O método COW, introduzido por Nielsen et al., ${ }^{10}$ é o algoritmo mais popular e eficiente no processo de alinhamento de picos cromatográficos, espectroscópicos e de ressonância magnética nuclear. ${ }^{17}$

Desta forma, um dos objetivos deste trabalho foi pré-tratar os perfís cromatográficos de voláteis de café torrado, alinhando-os pelo método COW, para então diferenciar as amostras segundo os três atributos sensoriais considerados: aroma, bebida e qualidade global. Neste caso, os cromatogramas devidamente pré-tratados foram interpretados diretamente na forma de perfis, e não pelo método tradicional que considera as áreas dos picos detectados. Através das análises realizadas para cada atributo estudado foi possível identificar compostos voláteis (marcadores) relacionados a cada um deles. Os resultados obtidos para cada atributo sensorial foram validados pelas seguintes figuras de mérito: sensitividade e especificidade.

\section{PARTE EXPERIMENTAL}

Amostras de café e análises sensoriais

Diversas amostras de café arábica cru de diferentes variedades

*e-mail: marcia@iqm.unicamp.br 
foram fornecidas pelo Centro de Análise e Pesquisa Tecnológica do Agronegócio do Café "Alcides Carvalho", situado no Instituto Agronômico de Campinas (IAC), e por cooperativas associadas dos Estados de São Paulo e Minas Gerais. Devido à classificação brasileira dos grãos por tipo, os defeitos encontrados nas amostras foram mantidos. Estas amostras foram degustadas por provadores e classificadas quanto ao aroma, sabor (bebida) e qualidade global.

Todas as notas atribuídas a cada parâmetro foram computadas, em escala de 1 a 5 de acordo com a magnitude da característica sensorial percebida, a partir da Tabela 1 fornecida como modelo para cada grupo de provadores.

Tabela 1. Escala numérica dos atributos fornecidos aos provadores para a realização das análises sensoriais

\begin{tabular}{cccc}
\hline Notas & Aroma & Bebida & Qualidade Global \\
\hline 1 & $\begin{array}{c}\text { Muito fraco ou } \\
\text { indesejável }\end{array}$ & Rio & Muito ruim \\
1,5 & M. fraco/fraco & Rio/Riada & M. ruim/ruim \\
2 & Fraco & Riada & Ruim \\
2,5 & Fraco/reg. & Dura - & Ruim/reg. \\
3 & Regular & Dura & Regular \\
3,5 & Reg./bom & Dura + & Reg./bom \\
4 & Bom & Apenas mole & Bom \\
4,5 & Bom/excel. & Mole & Bom/excel. \\
5 & Excelente & Estritamente mole & Excelente \\
\hline
\end{tabular}

De acordo com a Tabela 1, a nota do atributo bebida, por exemplo, veio acompanhada de uma classificação convencional brasileira. Esta classificação vai de café estritamente mole (nota 5 - excelente bebida) até café rio/riada (nota 1 - bebida ruim).

O preparo das amostras foi realizado utilizando-se a Portaria No 49 do Ministério de Agricultura, Pecuária e Abastecimento, de 25/03/2008 (http://www.agricultura.gov.br). A determinação do ponto de torra clara foi realizada por meio do Sistema Agtron (disco $\mathrm{n}^{\circ} 75$ ) e as infusões foram preparadas em xícara ou tigela pequena contendo $10 \mathrm{~g}$ de pó em $100 \mathrm{~mL}$ de água mineral quente ao ponto da primeira fervura.

Dentre todas as amostras analisadas sensorialmente, as bebidas de onze delas foram classificadas como de aroma fraco (notas entre 1-1,5), as bebidas de dez foram classificadas como riada ou rio (nota $1-1,5)$ e de 12 foram classificadas como de qualidade global baixa (nota 1-1,5). Vinte e duas amostras proporcionaram bebidas de aroma considerado pelos provadores como excelente (notas entre 4-5), 17 forneceram bebida mole (notas entre 4-5) e 24 foram consideradas como bebida de excelente qualidade global (notas entre 4-5).

Para as análises dos voláteis do café torrado por SPME-GC (solid phase microextraction - gas chromatography), $300 \mathrm{~g}$ de cada amostra de café arábica foram submetidos à torra clara/média (Sistema Agtron - disco n ${ }^{\circ} 75$ ) em torrador rotativo a gás da marca Pinhalense S/A Máquinas Agrícolas. O tempo médio de torra das amostras variou entorno de 12 min devido a diversos fatores, tais como, temperatura da chama, umidade da amostra, tamanho dos grãos. Os grãos torrados foram moídos em granulometria média e armazenados em embalagens metalizadas de alumínio recoberto por camadas plásticas de polietileno e poliestireno, seladas e armazenadas a $-5^{\circ} \mathrm{C}$ até a análise cromatográfica. Todo este cuidado foi tomado para diminuir a perda de voláteis na hora da abertura das embalagens. O intervalo de tempo entre a torra e a análise cromatográfica foi de no máximo $4 \mathrm{~h}$.

\section{Fibra de SPME}

A fibra de SPME empregada para a extração dos aromas foi a fibra comercial recoberta com polidimetilsiloxano/divinilbenzeno (PDMS/DVB) com $65 \mu \mathrm{m}$ de espessura $\left(V_{f}=0.357 \mathrm{~mm}^{3}\right)$ e o holder manual foram adquiridos da Supelco (Bellefonte, PA, EUA). Antes do seu uso, a fibra foi condicionada em cromatógrafo a gás por $12 \mathrm{~h}$ a $240{ }^{\circ} \mathrm{C}$, de acordo com instruções do manual da fibra (T7941231, Supelco Co., Bellefonte, PA, EUA).

\section{Análise por cromatografia a gás com detector em chama (CG- DIC)}

O modelo de cromatógrafo utilizado para as análises dos voláteis das amostras de café torrado foi o G-6850 serie GC system (Agillent, Wilmington, DE), com detecção por ionização em chama (DIC) e injetor com divisor/sem divisor de fluxo. A coluna cromatográfica utilizada foi a HP-5 (5\% difenilsiloxano e 95\% dimetilsiloxano; $30 \mathrm{~m}$ x 0,25 mm x 0,25 $\mu \mathrm{m}$ ). Hélio, a uma vazão de $1 \mathrm{~mL} \mathrm{~min}^{-1}$, foi utilizado como gás de arraste. $\mathrm{O}$ injetor foi equipado com um liner de diâmetro interno de $0,75 \mu \mathrm{m}$ e mantido a $220^{\circ} \mathrm{C}$ no modo sem divisor de fluxo. A temperatura do forno foi programada da seguinte maneira: de $40^{\circ} \mathrm{C}$ para $150{ }^{\circ} \mathrm{C}$, aumentando $7^{\circ} \mathrm{C}$ por minuto, seguido de aumento de $30{ }^{\circ} \mathrm{C}$ por minuto de $150{ }^{\circ} \mathrm{C}$ até $260^{\circ} \mathrm{C}$. Sob essas condições, não foi observado efeito de memória nas corridas em branco entre as extrações.

Para os procedimentos de extração dos voláteis, $250 \mathrm{mg}$ de café torrado e moído e $2 \mathrm{~mL}$ de solução saturada de cloreto de sódio foram colocados em recipientes de vidro de $5 \mathrm{~mL}$ de volume, com tampas perfuradas para adaptação de septos de silicone (Supelco). Os recipientes foram fechados e agitados a $900 \mathrm{rpm}$ com barras magnéticas. As condições experimentais utilizadas na extração dos voláteis dos cafés foram definidas de acordo com os resultados obtidos do planejamento experimental descrito por Ribeiro et al.:18 temperatura do banho $(T) 42,5^{\circ} \mathrm{C}, 10$ min de tempo de pré-equilíbrio $(T P E)$ e 22 min de tempo de extração $(T E)$. Todas as análises foram realizadas em triplicata.

\section{Análise por cromatografia a gás acoplada ao espectrômetro de massas (CG/EM)}

A identificação dos analitos extraídos foi realizada em cromatógrafo a gás modelo HP-5890 (Hewlett-Packard, Wilmington, DE, USA) operando com a mesma coluna utilizada no CG-DIC e equipado com detector de massas modelo HP-5973. Foram utilizadas também as mesmas condições analíticas empregadas no CG-DIC. A faixa de aquisição dos espectros foi de $40-350 \mathrm{~m} / \mathrm{z}$. No tratamento dos dados obtidos pelo GC-MS, utilizou-se o software Automated Mass Spectral Deconvolution and Identification System (AMDIS) v. 2.61 e o NIST Mass Spectral Search Program v. 2.0d (Washington, DC, USA). Comparações foram feitas também com trabalhos encontrados na literatura sobre voláteis de café torrado identificados por espectrometria de massas. ${ }^{4,19,20} \mathrm{~A}$ partir da análise cromatográfica da série homóloga de alcanos $\left(\mathrm{C}_{4}-\mathrm{C}_{14}\right)$ foram calculados os índices de retenção (IRc) dos compostos voláteis segundo van der Dool e Kratz. ${ }^{21}$

\section{Quimiometria}

Todos os dados cromatográficos obtidos foram organizados na forma de matrizes $\mathbf{X}(I \mathrm{x} J)$ contendo $I$ linhas (amostras) e $J$ colunas (variáveis), nas quais cada replicata foi considerada como uma amostra. As $J$ variáveis, neste caso, foram as leituras (intensidade do sinal em picoampére - $\mathrm{pA}$ ) realizadas pelo detector utilizado (DIC) 
a cada $65 \mathrm{~ms}$ de corrida cromatográfica. Para o processamento dos dados empregou-se o software Mathlab 6.5 (The MathWorks, Co., Natick, MA, USA) e o pacote computacional PLS_Toolbox (Eigenvector Research, Inc. - PLS_Toolbox version 3.02.).22 A análise de componentes principais (PCA - Principal Component Analysis) foi utilizada como método exploratório. ${ }^{23}$

Os dados cromatográficos foram alinhados utilizando o algoritmo $\mathrm{COW}^{10}$ disponível na página www.models.kvl.dk/source/. Os cromatogramas foram divididos em 5 partes e cada uma das partes foi alinhada utilizando $N=10$ e $s=1$. Após o alinhamento, os cromatogramas foram normalizados pela norma Euclidiana $\left(I_{2}\right)$, fazendo com que o comprimento de cada um deles seja igual a 1. Posteriormente, a $1^{\text {a }}$ derivada foi aplicada e os dados foram autoescalados. ${ }^{24}$ A seleção das variáveis (intensidades dos picos cromatográficos) para essas análises foi realizada visualmente, através da sobreposição de todos os cromatogramas e observação das diferenças sistemáticas entre as amostras.

O algoritmo COW alinha um cromatograma desalinhado e representado por $\mathbf{x}_{d}$ a um cromatograma de referência designado por $\mathbf{x}_{r}$. Neste caso, $\mathbf{x}_{r}$ pode ser o cromatograma médio, calculado tomando-se a média das colunas de todos os cromatogramas que serão alinhados, ou aquele que apresentar, visualmente falando, o melhor sinal como por exemplo, o cromatograma com os picos mais resolvidos. Considerando $\mathbf{x}_{d}$ e $\mathbf{x}_{r}$ como vetores contendo $J$ e $J$ ' coordenadas, respectivamente ( $J$ e $J$ ' são as variáveis ou leituras do detector para os tempos de retenção), $\mathbf{x}_{d}$ será alinhado a $\mathbf{x}_{r}$ por meio de compressões e estiramentos (deformações) em seções previamente definidas do cromatograma. A solução geral para o alinhamento corresponderá a uma combinação das diversas deformações para cada seção, que fornecerá a máxima correlação entre $\mathbf{x}_{d}$ e $\mathbf{x}_{r}$, aproximando o máximo possível o vetor desalinhado do vetor de referência.

$\mathrm{O}$ algoritmo funciona da seguinte forma:

1. tanto o vetor a ser alinhado, $\mathbf{x}_{d}$, quanto o vetor de referência, $\mathbf{x}_{r}$, são divididos em um número $N$ de seções, especificadas pelo analista (Equação 1). Cada seção tem comprimento $M$ e $M^{\prime}$ em $\mathbf{x}_{d}$ e $\mathbf{x}_{r}$, respectivamente, que neste contexto significa o número de variáveis das seções, aqui denominadas, g e g'. Além disso, são definidos para cada seção os "nós" que efetivamente sofrerão deslocamentos e proporcionarão as deformações.

$$
N=J / M
$$

2. cada seção $\mathbf{g}_{n}(n=1,2, \ldots, N)$, de comprimento $M$, é distorcida, tendo seu comprimento alongado ou encurtado por um número prédefinido de pontos, i. e., variando o número de coordenadas do vetor $\mathbf{x}_{d}$, que por sua vez são obtidos através de interpolação linear entre apropriados pontos adjacentes entre $\mathbf{x}_{d}$ e $\mathbf{x}_{r}$. A variação corresponde ao deslocamento dos "nós" mediante uma amplitude máxima prédefinida. No caso de $\mathbf{x}_{d}$ e $\mathbf{x}_{r}$ possuírem o mesmo número de coordenadas, $J$ será igual a $J$ ' e $M$ será igual a $M^{\prime}$, havendo então apenas um deslocamento da seção. Vale ressaltar que no alinhamento, os pontos iniciais e finais (primeiro e último nós) do vetor desalinhado $\mathbf{x}_{d}$ e do vetor de referência $\mathbf{x}_{r}$ são sempre igualados e permanecem fixos durante todo o procedimento.

Diante do exposto acima, fica clara a necessidade de definir dois parâmetros que são responsáveis pela qualidade do alinhamento. O primeiro deles é o número de seções $N\left(\mathbf{g}_{n} n=1,2, \ldots\right.$, $N)$ no qual o cromatograma é dividido, definindo a posição dos nós. O segundo parâmetro, $s$, mais conhecido como parâmetro de distorção (slack), define todas as possíveis deformações (encurtamento ou alongamento), ou seja, as posições finais dos nós de uma seção de comprimento $M$. A qualidade do alinhamento está diretamente ligada à razão entre esses parâmetros (slack/compri- mento das seções), que pode ser entendida como a flexibilidade do alinhamento. Desse modo, o que importa é esta razão e não o valor numérico dos parâmetros em si.

A posição dos pontos de extremidade (nós) de cada seção $\mathbf{g}_{n}$ é definida pelo parâmetro $s$. Então, cada seção pode ser alongada ou encurtada de acordo com a amplitude definida pelo parâmetro de distorção, de $-s$ a $+s$ pontos, com exceção dos pontos inicial $\left(x_{d(i, 1)}\right) \mathrm{e}$ final $\left(x_{d(i, J)}\right)$ dos vetores que são fixados. Por exemplo, se $s$ for igual a dois, existem cinco possíveis pontos finais de uma seção: $-2,-1$, 0,1 e 2. Logo, estes cinco finais possíveis, obtidos para cada $\mathbf{g}_{n}$ são linearmente interpolados à respectiva seção $\mathbf{g}_{n}^{\prime}$. Desta forma, coeficientes de correlação $(r)$ entre os 5 possíveis $\mathbf{g}_{\mathrm{n}}$ e $\mathbf{g}_{n}$ são calculados, e o alinhamento que apresentar a melhor correlação, ou seja, o melhor alinhamento da seção é escolhido. O processo é repetido para as seções $\mathbf{g}_{n+1}$ e $\mathbf{g}_{n+1}$ e o melhor alinhamento é armazenado e, assim, sucessivamente para todas as $N$ seções.

Por fim, a melhor solução de distorção (o melhor alinhamento) do vetor $\mathbf{x}_{d}$ é construída a partir da união das $N$ seções que apresentam os melhores coeficientes de correlação $r$. Ou seja, à posição do ponto final da primeira seção $\left(\mathbf{g}_{1}\right)$ em $\mathbf{x}_{d}$ (definida por um dado valor de $s$ ) une-se o ponto inicial da segunda seção de $\mathbf{x}_{d}\left(\mathbf{g}_{2}\right)$ e assim por diante.

O tempo computacional do método COW aumenta exponencialmente com os valores de $s$. A seleção deste parâmetro é guiada geralmente por deslocamentos observados entre os picos dos vetores $\left(\mathbf{x}_{r}\right.$ e $\left.\mathbf{x}_{d}\right)$ e pelo próprio comprimento da seção. O parâmetro $s$ deve ser grande o suficiente para assegurar a flexibilidade satisfatória de distorção, sendo que o número de variáveis em uma seção subtraído de $s$ deve ser maior que 1. Entretanto, para diminuir este tempo computacional e aumentar a flexibilidade no alinhamento, uma alternativa seria aumentar o número de seções mantendo baixos valores de $s .{ }^{17}$

Conforme já foi mostrado, uma restrição do algoritmo COW é que os pontos nas extremidades do vetor desalinhado e do vetor referência são igualados e fixos, o que possibilita também igualar sinais processados de diferentes comprimentos ( $J$ diferente de $J$ '). Se cada seção ( $\left.\mathbf{g}_{n}\right)$ de $\mathbf{x}_{d}$ for interpolada linearmente ao comprimento de uma seção $\left(\mathbf{g}_{n}{ }^{\prime}\right)$ em $\mathbf{x}_{r}$, têm-se após o alinhamento sinais de mesmo comprimento.

Como os pontos inicial e final de um cromatograma são fixos, a flexibilidade de distorção para seções localizadas mais ao centro é maior, uma vez que há uma maior distância das restrições. Como consequência, é mais difícil alinhar picos que estão no início ou na extremidade do sinal. ${ }^{25}$

\section{Figuras de mérito}

Neste artigo duas figuras de mérito, a sensitividade e a especificidade, foram calculadas e utilizadas na análise dos dados descritos. ${ }^{26}$ Para a obtenção destas figuras de mérito, uma análise de componentes principais é realizada para duas ou mais classes pré-determinadas de amostras $(A, B, \ldots, Z)$ e a matriz de erros é calculada de acordo com a Equação 2:

$$
\mathbf{E}=\mathbf{X}-\mathbf{S}_{k} \mathbf{P}_{k}^{\mathrm{T}}
$$

onde $k$ é o número apropriado de componentes principais utilizadas para descrever o conjunto de dados e $\mathbf{S}_{k}(\mathrm{Ix} k)$ e $\mathbf{P}_{\boldsymbol{k}}(J \mathrm{x} k)$ são, respectivamente, as matrizes de escores e pesos truncadas em $k$, e $\mathbf{E}(I \mathrm{x} J)$ é a matriz de resíduos cujos elementos são representados por $e_{i j}$.

Feitas essas considerações, pode-se obter as matrizes de resíduos para ambas as classes, o desvio padrão residual de cada objeto $i$ em sua respectiva classe e o desvio padrão residual total de cada classe. Para a classe $A$, o desvio padrão residual do objeto $i, \mathrm{~S}_{i}^{A}$, e o desvio padrão residual total, $\mathrm{S}_{0}{ }^{A}$, são calculados de acordo com a Equação 3 . 


$$
\mathrm{s}_{i}^{A}=\sqrt{\frac{\sum_{j=1}^{J}\left(e_{i j}^{A}\right)^{2}}{\left(J-k_{A}\right)}} \mathrm{e}_{\mathrm{s}_{0}^{A}}=\sqrt{\frac{\sum_{i=1}^{I_{A}} \sum_{j=1}^{J}\left(e_{i j}^{A}\right)^{2}}{\left(I_{A}-k_{A}-1\right)\left(J-k_{A}\right)}}
$$

A classificação dos objetos em uma das classes $A$ ou $B$ pode ser visualizada em um gráfico de Coomans $^{27}$ cujos eixos correspondem aos desvios padrão $\mathrm{S}_{i}^{A}$ e $\mathrm{S}_{i}^{B}$. Isto é viável porque estes dois parâmetros representam a distância dos diversos objetos (amostras) ao hiperplano definido pelas componentes principais $k_{A} e k_{B}$ das classes $A$ e $B$, respectivamente. Espera-se que para as amostras pertencentes à classe $A$, a razão entre as variâncias $\left(\mathrm{S}_{i}^{A}\right)^{2} /\left(\mathrm{S}_{0}{ }^{A}\right)^{2}$ siga uma distribuição $F$ com $\left(J-k_{A}\right)$ e $\left(I_{A}-k_{A}-1\right)\left(J-k_{A}\right)$ graus de liberdade. Com este teste, tem-se uma base estatística para decidir quantitativamente se uma amostra está muito distante do modelo ou não. Para um determinado nível de significância $\alpha$, obtém-se o valor tabelado $\mathrm{F}_{\text {crítico }}$ que é o valor máximo de $F$ para que uma amostra seja classificada nesta classe. A partir daí, determina-se um valor de corte, $\mathrm{S}_{\text {crit }}^{A}$, para a classe $A, \mathrm{~S}_{\text {crit }}^{A}=\mathrm{S}_{0} \sqrt{ } F_{\text {crit }}$. O mesmo é feito para a classe $B$.

Observando o exemplo indicado na Figura 1, há 22 amostras pertencentes à classe $A(\bullet, \bigcirc, \bullet)$ e 27 à classe $B(\square, \square, \square)$. A linha tracejada vertical é a linha de corte, com $95 \%$ de probabilidade $(\alpha=0,05)$, para classe $A$ e a linha tracejada horizontal idem para a classe $B$. As amostras localizadas no $2^{\circ}$ quadrante, $2^{\circ} \mathrm{Q}$, pertencem exclusivamente à classe $A(\bullet)$ e foram reconhecidas pelo modelo (hiperplano) construído para esta classe. $\mathrm{O}$ mesmo acontece com as amostras no $4^{\circ}$ quadrante, porém, pertencentes à classe $B(\square)$. Já as amostras localizadas no $3^{\circ}$ quadrante $(\square, \bigcirc)$ são pertencentes a uma das duas classes, mas reconhecidas por ambas. Por fim, as amostras localizadas no $1^{\circ}$ quadrante $(\boldsymbol{\square}, \bullet)$ pertencem a uma das classes, porém, não foram reconhecidas por nenhum dos modelos construídos para elas. Em alguns casos, as amostras encontradas no $1^{\circ}$ quadrante podem ser outliers ou amostras pertencentes a outras classes que não foram modeladas. Na Figura 1 estão incluídas, ainda, as distâncias de uma amostra $a_{i}$ que pertence à classe $A$. Esta amostra está próxima da classe $A$ e distante da $B$.

Através da localização das amostras no gráfico de distâncias de Coomans, pode-se calcular a sensitividade e a especificidade de cada classe.

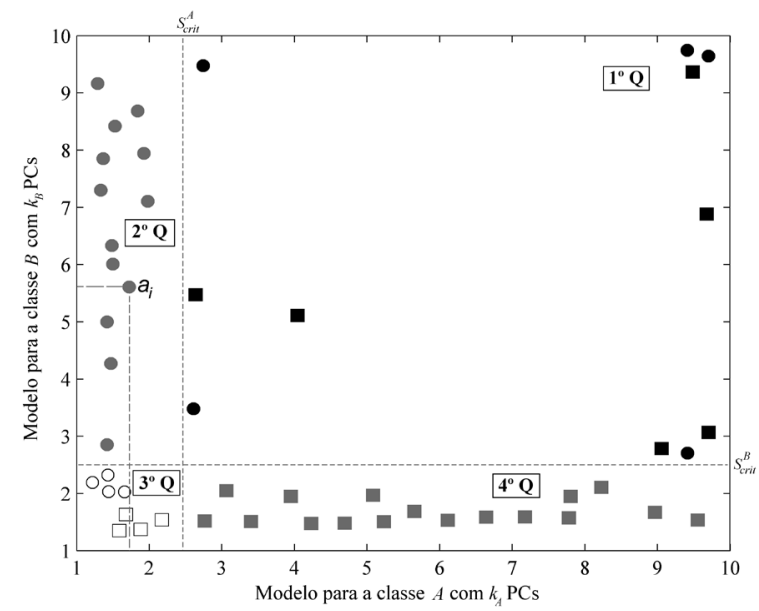

Figura 1. Gráfico de Coomans para duas classes de amostras (A e B). Amostras representadas por círculo são da classe $A$ e as representadas por quadrados pertencem à classe B. Amostras no $1^{\circ}$ quadrante foram classificadas como não pertencentes a nenhuma das classes e as do $3^{\circ}$ quadrante foram classificadas como pertencentes a ambas as classes

Assim, a sensitividade para o modelo da classe $A$, por exemplo, é calculada como a porcentagem de amostras pertencentes a esta classe e que foram corretamente classificadas. Neste caso, é a razão entre o $\mathrm{n}^{\circ}$ de amostras reconhecidas pelo modelo $(\bullet, \bigcirc)$ e o $\mathrm{n}^{\circ}$ total de amostras da classe $A(\bullet, \bigcirc, \bullet)$. Já a especificidade (ou seletividade) do modelo da classe $A$ é a porcentagem de amostras não pertencentes à classe $A$ e que foram corretamente consideradas como sendo desta outra classe $(B)$ ou não reconhecida por ela $(B)$. Neste caso, é a razão entre as amostras reconhecidas e pertencentes à classe $B(\square, \square)$ e todas as amostras da classe $B(\square, \square, \square)$.

\section{RESULTADOS E DISCUSSÃO}

Neste trabalho foram realizadas três análises de componentes principais distintas, sendo uma para cada um dos atributos sensoriais, aroma, bebida e qualidade global.

\section{Análise dos voláteis por espectrometria de massas}

Pela análise por espectrometria de massas foram detectados mais de 250 compostos, dos quais 54 se mostraram importantes para uma ou mais das análises de componentes principais. A Tabela 2 mostra estes compostos com seus respectivos tempos de retenção, nomenclatura, fragmentação $(\mathrm{m} / \mathrm{z}$ dos íons mais intensos - abundância relativa $\%)$, math (índice de similaridade entre 2 espectros de massas) e em qual das análises quimiométricas foram utilizados.

\section{Análise de componentes principais para o atributo aroma}

Para a classificação segundo o aroma, foram selecionadas 11 amostras com notas baixas, ou seja, com característica de aroma fraco ou atípico (classe 1), e 22 amostras apresentando notas elevadas nesse atributo (classe 2). Para reduzir as informações irrelevantes, que poderiam mascarar dos resultados da PCA, foi feita uma seleção visual de variáveis, mediante a sobreposição de todos os cromatogramas. Por essa técnica, a matriz original $\mathbf{X}_{p}$ (33 x 20640) dos dados pré-tratados, contendo todos os picos mais a linha de base, foi reduzida para (33 x 2393), correspondendo a apenas 39 picos. Esses picos estão indicados pelas linhas verticais na Figura 2, que apresenta um cromatograma característico de voláteis de café arábica. Os números indicados sobre cada pico na Figura 2 correspondem aos compostos descritos previamente na Tabela 2 .

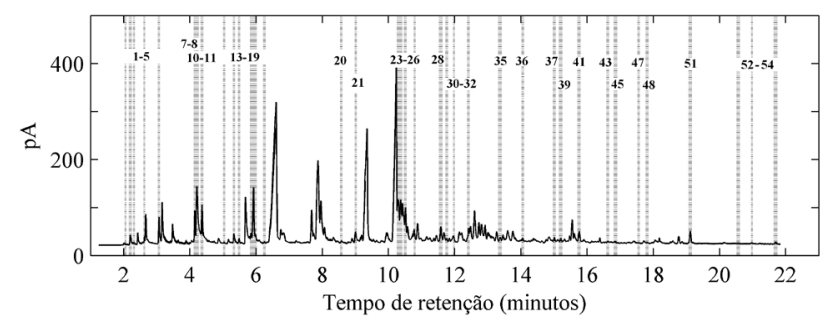

Figura 2. Picos selecionados para a PCA de aroma (A). O cromatograma é típico de voláteis de café arábica torrado extraídos com a fibra de PDMS/ DVB 65 um e separados por cromatografia gasosa

Utilizando os 39 picos selecionados, a PCA descreveu 46,96\% da variância total dos dados selecionados com apenas 2 componentes principais. A porcentagem de variância que cada componente descreve está detalhada no gráfico de escores e pesos (Figura 3). Esta análise revelou que a separação entre as duas classes está principalmente relacionada à primeira componente principal ( $\mathrm{PC} 1)$, em que as amostras da classe 1 (aroma baixo) (•) se encontram à esquerda desta componente, relativamente separadas das amostras da classe 
Tabela 2. Compostos voláteis utilizados em uma ou mais análise de componentes principais e identificados por comparação dos seus espectros de massas com os do banco de dados do NIST e da literatura

\begin{tabular}{|c|c|c|c|c|c|c|c|}
\hline $\mathrm{N}^{\circ}$ comp. & $\mathrm{t}_{\mathrm{R}}(\min )$ & $\mathrm{IR}_{\mathrm{c}}$ & $\mathrm{IR}_{1}$ & Nome do composto & Íons característicos $(\mathrm{m} / \mathrm{z})^{\mathbf{b}}$ & Math & $\mathrm{PCA}^{\mathrm{c}}$ \\
\hline 1 & 2.05 & 493 & 500 & Metanotiol & 47 (B), 45,60 & 986 & $\mathrm{~A}, \mathrm{~B}$ \\
\hline 2 & 2.2 & 521 & 477 & Acetona & 43 (B), 58 & 870 & $\mathrm{~A}, \mathrm{C}$ \\
\hline 3 & 2.3 & 536 & 515 & Acetato de metila & 43 (B), 74 & 944 & $\mathrm{~A}, \mathrm{~B}$ \\
\hline 4 & 2.625 & 587 & 601 & 2-Butanona & 43 (B), 73 & 959 & $\mathrm{~A}, \mathrm{~B}$ \\
\hline 5 & 3.065 & 642 & 648 & 3-metilbutanal & 41 (B), 39, 58, 71 & 962 & A \\
\hline 6 & 3.475 & 685 & 701 & 2,3-Pentanodiona & 43 (B), 57 & 945 & $\mathrm{~B}, \mathrm{C}$ \\
\hline 7 & 4.15 & 747 & - & Pirrol & 67 (B), 39 & 954 & $\mathrm{~A}, \mathrm{~B}, \mathrm{C}$ \\
\hline 8 & 4.185 & 750 & - & $\mathrm{N} / \mathrm{I}^{\mathrm{a}}$ & - & - & A \\
\hline 9 & 4.22 & 749 & 753 & Piridina & 79 (B), 52 & 936 & $\mathrm{~B}, \mathrm{C}$ \\
\hline 10 & 4.249 & 754 & - & $\mathrm{N} / \mathrm{I}$ & - & - & A \\
\hline 11 & 4.365 & 759 & 749 & Metilpirrol & 81 (B), 39, 42, 53 & 942 & $\mathrm{~A}, \mathrm{~B}, \mathrm{C}$ \\
\hline 12 & 4.87 & 782 & 733 & 2,3- Hexadiona & 43 (B), 71,114 & 911 & $\mathrm{C}$ \\
\hline 13 & 5.03 & 790 & 767 & Ciclopentanona & & & $\mathrm{A}, \mathrm{B}, \mathrm{C}$ \\
\hline 14 & 5.325 & 803 & - & Di-hidro-2-metil-3-furanona & 43 (B), 72, 100 & 928 & $\mathrm{~A}, \mathrm{~B}, \mathrm{C}$ \\
\hline 15 & 5.483 & 810 & 820 & Etilpirrol & 80 (B), 95, 67, 53, 78 & 806 & A \\
\hline 16 & 5.85 & 826 & - & $\mathrm{N} / \mathrm{I}$ & - & - & $\mathrm{A}, \mathrm{B}$ \\
\hline 17 & 5.92 & 832 & 830 & Furfural & 39 (B), 95 & 953 & $\mathrm{~A}, \mathrm{~B}, \mathrm{C}$ \\
\hline 18 & 5.98 & 832 & - & 2,N-dimetilpirrol & 94 (B), 42 & 800 & A \\
\hline 19 & 6.25 & 842 & 857 & Trimetiloxazol & 111 (B), 42, 55, 68 & 882 & A \\
\hline 20 & 8.58 & 934 & - & Etenilpirazina & 106 (B), 52, 79 & 820 & A, B \\
\hline 21 & 9 & 950 & 959 & 3-etilpiridina & 92 (B), 107, 65, 39 & 918 & $\mathrm{~A}, \mathrm{C}$ \\
\hline 22 & 9.34 & 963 & - & 5-metil-2-furancarboxaldeído & $53(\mathrm{~B}), 110,81$ & 938 & $\mathrm{~B}, \mathrm{C}$ \\
\hline 23 & 10.29 & 998 & 1001 & 2-etil-6-metilpirazina & 121 (B), 94, 128 & 830 & $\mathrm{~A}, \mathrm{C}$ \\
\hline 24 & 10.37 & 1001 & - & 2-etil-5-metilpirazina & 121 (B), 39, 58 & 793 & $\mathrm{~A}, \mathrm{~B}, \mathrm{C}$ \\
\hline 25 & 10.505 & 1010 & - & 1-metilpirrol 2-carboxaldeído & 109 (B), 53, 39, 80 & 859 & A \\
\hline 26 & 10.77 & 1021 & - & 2-etenil-n-metilpirazina & 52 (B), 120, 39 & 778 & $\mathrm{~A}, \mathrm{~B}, \mathrm{C}$ \\
\hline 27 & 11.25 & 1041 & - & 2-acetilpiridina & 79 (B), 43, 121 & 847 & $\mathrm{C}$ \\
\hline 28 & 11.57 & 1055 & - & Benzenoacetaldeído & 91 (B), 120, 65, 39 & 893 & A, B \\
\hline 29 & 11.675 & 1059 & - & 4-piridazinamida & 95 (B), 43 & 748 & $\mathrm{C}$ \\
\hline 30 & 11.75 & 1062 & - & 1-(2'-furil)-2-butanona & 57 (B), 81, 138 & 840 & $\mathrm{~A}, \mathrm{~B}$ \\
\hline 31 & 11.96 & 1071 & - & $\mathrm{N} / \mathrm{I}$ & - & - & $\mathrm{A}, \mathrm{B}, \mathrm{C}$ \\
\hline 32 & 12.415 & 1090 & 1072 & 2-acetilpirrol & 94 (B), 66, 109, 43 & 890 & A \\
\hline 33 & 12.475 & 1093 & - & 3-etil-2,5-dimetilpirazina & 42 (B), 39, 135 & 905 & $\mathrm{C}$ \\
\hline 34 & 13.26 & 1126 & - & $\mathrm{N} / \mathrm{I}$ & - & - & $\mathrm{B}$ \\
\hline 35 & 13.37 & 1129 & - & $\mathrm{N} / \mathrm{I}$ & - & - & A \\
\hline 36 & 14.05 & 1159 & - & 5-metil-2-propionilfurano & 109 (B), 53, 138 & 876 & $\mathrm{~A}, \mathrm{~B}, \mathrm{C}$ \\
\hline 37 & 15.01 & 1200 & - & $\mathrm{N} / \mathrm{I}$ & - & - & A, B \\
\hline 38 & 15.02 & 1200 & - & $\mathrm{N} / \mathrm{I}$ & - & - & $\mathrm{B}$ \\
\hline 39 & 15.205 & 1208 & - & $\mathrm{N} / \mathrm{I}$ & - & - & $\mathrm{A}, \mathrm{C}$ \\
\hline 40 & 15.61 & 1225 & - & Coeluição - N/I & - & - & $\mathrm{B}, \mathrm{C}$ \\
\hline 41 & 15.76 & 1232 & - & Coeluição - N/I & - & - & $\mathrm{A}, \mathrm{B}, \mathrm{C}$ \\
\hline 42 & 16.145 & 1248 & - & $\mathrm{N} / \mathrm{I}$ & - & - & $\mathrm{C}$ \\
\hline 43 & 16.385 & 1258 & - & Furfurildissulfeto & 81 (B), 53, 160 & 891 & $\mathrm{~A}, \mathrm{C}$ \\
\hline 44 & 16.63 & 1266 & 1269 & Pentanoato de furfurila & 81 (B), 98, 182 & 784 & $\mathrm{~B}, \mathrm{C}$ \\
\hline 45 & 16.845 & 1273 & - & $\mathrm{N} / \mathrm{I}$ & - & - & $\mathrm{A}, \mathrm{B}, \mathrm{C}$ \\
\hline 46 & 17.41 & 1284 & - & $\mathrm{N} / \mathrm{I}$ & - & - & $\mathrm{B}$ \\
\hline
\end{tabular}


Tabela 2. continuação

\begin{tabular}{|c|c|c|c|c|c|c|c|}
\hline $\mathrm{N}^{\circ}$ comp. & $\mathrm{t}_{\mathrm{R}}(\min )$ & $\mathrm{IR}_{\mathrm{c}}$ & $\mathrm{IR}_{1}$ & Nome do composto & Íons característicos $(\mathrm{m} / \mathrm{z})^{\mathbf{b}}$ & Math & $\mathrm{PCA}^{\mathrm{c}}$ \\
\hline 47 & 17.56 & 1286 & - & $\mathrm{N} / \mathrm{I}$ & - & - & A \\
\hline 48 & 17.81 & 1291 & - & N/I & - & - & $\mathrm{A}, \mathrm{B}, \mathrm{C}$ \\
\hline 49 & 18.055 & 1296 & - & Furfurilmetildissulfeto & 81 (B), 53, 161 & 793 & $\mathrm{C}$ \\
\hline 50 & 18.525 & 1305 & - & $\mathrm{N} / \mathrm{I}$ & - & - & $\mathrm{B}, \mathrm{C}$ \\
\hline 51 & 19.115 & 1314 & 1317 & 4-vinilguaiacol & 77 (B), 135, 150, 107, 51 & 907 & A \\
\hline 52 & 20.57 & 1330 & - & $\mathrm{N} / \mathrm{I}$ & - & - & A \\
\hline 53 & 20.96 & 1337 & - & $\mathrm{N} / \mathrm{I}$ & - & - & A \\
\hline 54 & 21.7 & 1349 & - & $\mathrm{N} / \mathrm{I}$ & - & - & $\mathrm{A}, \mathrm{B}, \mathrm{C}$ \\
\hline
\end{tabular}

A. Aroma; $B$. Bebida; $C$. Qualidade global; a. N/I - Não identificados; b. $\mathrm{m} / z$ dos íons mais intensos; c. Picos usados nas análises dos atributos; B. Pico base; $\mathrm{t}_{\mathrm{R}}$. tempo de retenção. IR. Índice de retenção calculado; $\mathrm{IR}_{\mathrm{l}}$. Índice de retenção da literatura para colunas similares a HP-5. 28,29

2 (aroma excelente) $(\square)$ dispostas à direita de PC1. Os números em cinza e em preto indicados na Figura 3 representam os compostos voláteis (picos cromatográficos) selecionados e a influência (o peso) de cada um deles na separação das amostras.

Dentre os 39 picos selecionados para separar as amostras de acordo com o atributo aroma, 4 deles são citados na literatura como odorantes potentes do café torrado. ${ }^{30,31}$ São eles: metanotiol (1), 3-metilbutanal (5), 2,3-pentanodiona (6), 4-vinilguaiacol (51).

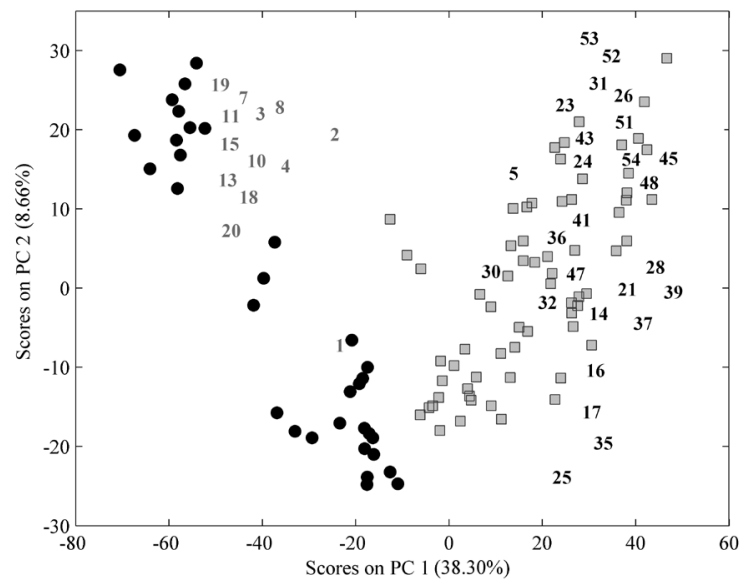

Figura 3. Gráfico de escores e pesos de PC1 versus PC2 para as amostras estudadas no atributo aroma (A) - Amostras com aroma fraco elou muito fraco (•) e amostras com excelente aroma ( $\square$ ). Os números em cinza e em preto representam o peso de cada pico entre as amostras das duas classes

\section{Análise de componentes principais para o atributo sabor (bebida)}

Para esta análise, foram utilizadas 10 amostras de café com bebida de características rio ou riada e 17 de bebidas classificadas sensorialmente como bebida mole. Trinta picos, correspondentes a 2415 variáveis das 20640 originais, foram selecionados visualmente. Esses picos (ou variáveis) estão indicados em linhas verticais na Figura 4 e enumerados de acordo com a Tabela 2.

No gráfico dos escores e pesos (Figuras 5), verificou-se uma tendência de agrupamento das amostras ao longo de PC1. Essa PC explicou $45,45 \%$ da variância dos picos selecionados. As amostras à esquerda de $\mathrm{PC} 1$ (rio ou riadas) formam um agrupamento $(\bullet)$, enquanto as amostras à direita (moles) $(\square)$ formam outro. Os números em cinza e em preto na Figura 5 representam os compostos selecionados e a influência de cada um na classificação das amostras.

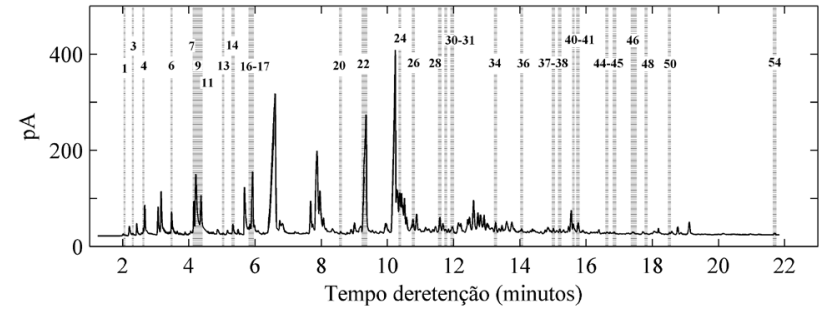

Figura 4. Picos selecionados para a análise PCA de bebida (B). O cromatograma é típico de voláteis de café arábica torrado extraídos com a fibra de PDMS/DVB 65 um e separados por cromatografia gasosa

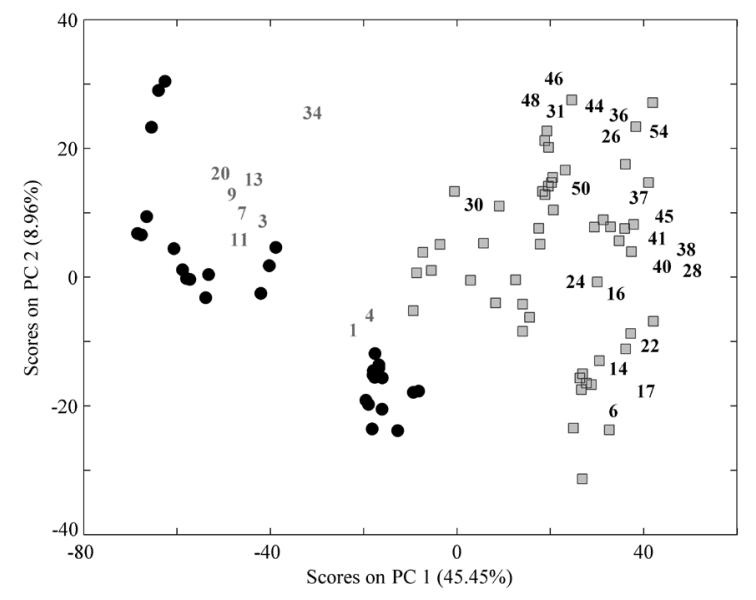

Figura 5. Gráfico de escores e pesos de PC1 versus $P C 2$ para as amostras estudadas no atributo bebida (B) - Amostras com bebida rio/riada (•) $e$ amostras com bebida mole $(\square)$. Os números em cinza e em preto representam o peso de cada pico entre as amostras

\section{Análise de componentes principais para a qualidade global}

Nesta análise, foram utilizadas 24 amostras com notas elevadas de qualidade global e 12 com notas baixas no atributo. Após a seleção de variáveis, 30 picos cromatográficos referentes a 2032 variáveis foram considerados na análise de PCA. A Figura 6 mostra um cromatograma típico dos voláteis de café arábica extraídos pela fibra de PDMS/DVB $65 \mu \mathrm{m}$, bem como os picos, enumerados de acordo com a Tabela $2 \mathrm{e}$ indicados em linhas verticais.

De acordo com o gráfico de escores e pesos de PC1 x PC2 (Figura 7), as amostras com bebidas de baixa qualidade global (•) estão localizadas na parte negativa de PC1, enquanto as amostras de alta 


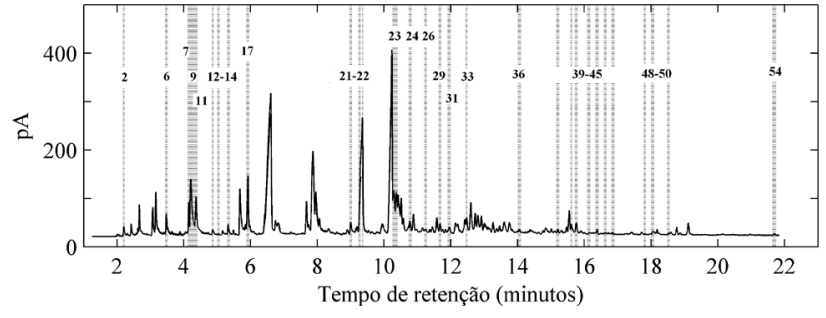

Figura 6. Picos selecionados para a PCA para qualidade global (C). $O$ cromatograma é típico de voláteis de café arábica torrado extraídos com a fibra de PDMS/DVB 65 um e separados por cromatografia gasosa

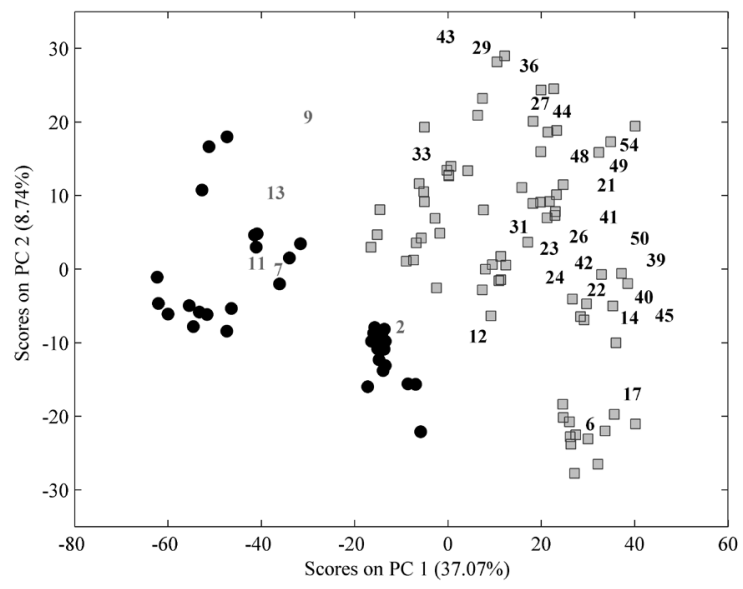

Figura 7. Gráfico de escores e pesos de PC1 versus PC2 para as amostras estudadas no atributo qualidade global (C) - Amostras com baixa qualidade global (○) e amostras com alta qualidade global ( $\square$ ). Os números em cinza e em preto representam o peso de cada pico entre as amostras

qualidade $(\square)$ tendem a ficar na parte positiva desta componente principal. A PC1 explicou 37,07\% da variância dos 30 picos selecionados.

De acordo com as análises de componentes principais realizadas para diferenciar bebidas de cafés segundo seus voláteis, os compostos pirrol (7), metilpirrol (11), ciclopentanona (13), di-hidro2-metil-3-furanona (14), furfural (17), 2-etil-5-metilpirazina (24), 2-etenil-n-metilpirazina (26), 5-metil-2-propionilfurano (36) e mais 5 compostos não identificados $(\mathbf{3 1}, \mathbf{4 1}, \mathbf{4 5}, \mathbf{4 8}, \mathbf{5 4})$ seriam possíveis marcadores tanto do aroma quanto do sabor e da qualidade global, simultaneamente. Destes, os compostos 7, 11 e 13 quando aparecem em maior concentração tendem a diminuir a qualidade das bebidas. Contrariamente, os outros compostos identificados aumentam a qualidade dos cafés quando em maior concentração.

Segundo Morais et al., ${ }^{32}$ a concentração relativa da 2-butanona (4) é maior em cafés torrados com presença de defeitos (PVA) comparada a cafés sem defeitos. Além da 2-butanona (4), o pirrol (7) e o metilpirrol (11) também aparecem em maior concentração nesses cafés (PVA), porém, a diferença é menos acentuada.

A análise efetuada para o atributo "qualidade global" confirma alguns dos resultados apresentados em Ribeiro et al., ${ }^{4}$ em que os compostos furfural (17) e 5-metil- 2-furancarboxaldeído (22) aparecem como possíveis marcadores desse atributo da bebida. O composto metilpirrol (11) foi identificado como marcador negativo de qualidade, confirmando o apresentado na literatura. ${ }^{32-34}$ Segundo Toci e Farah, ${ }^{7}$ a piridina (9) e o etenilpirazina (20) também indicam baixa qualidade de bebida. A piridina também é descrita como composto característico de torras escuras. ${ }^{35,36}$ As análises de componentes principais revelaram que esses compostos interferem no aroma e na bebida (20), bem como na bebida e na qualidade global (9) da bebida de café. Entretanto, o composto 2-etil-5-metilpirazina (24) neste trabalho foi identificado como marcador positivo para os 3 atributos sensoriais, mas apresentou caráter negativo no trabalho de Agresti et al.. ${ }^{6}$

Os outros compostos trazidos na Tabela 1 são igualmente possíveis marcadores de um ou mais dos três atributos estudados, com efeitos negativos ou positivos, conforme suas posições nos gráficos de escores e pesos.

\section{Figuras de mérito}

Para o cálculo da sensitividade e especificidade, foram feitas análises de componentes principais com os dados utilizados nos itens anteriores. Para isso, as amostras descritas na parte experimental foram organizadas em classes como indicado na Tabela 3, que contém o número de componentes principais utilizados para descrever cada classe e as respectivas distâncias críticas determinadas por um teste $F$ com $95 \%$ de significância.

Para o modelo para aroma, a sensitividade foi de $100 \%$ para as categorias de café de aroma bom e de aroma ruim, indicando que estas duas classes são capazes de identificar as amostras pertencentes a elas. Quanto à especificidade, a classe de cafés de aroma bom rejeitou apenas $45 \%$ das amostras de cafés de aroma ruim. Em outras palavras, a classe de cafés bons aceitou 55\% das amostras de aroma ruim. Por outro lado, a classe de cafés ruins se mostrou satisfatoriamente seletiva. Ela reconheceu todas as amostras de sua classe e $22 \%$ das amostras de aroma bom.

No caso do atributo bebida, a sensitividade foi de $100 \%$ para as duas categorias de café (rio/riada e mole), indicando que ambas são capazes de identificar as suas amostras. Quanto à especificidade, a classe de cafés moles rejeitou $90 \%$ das amostras de cafés rio/riados, enquanto que a classe de cafés rio/riados rejeitou $94 \%$ dos cafés moles, demonstrando alta especificidade em ambas. Devido a está alta especificidade das duas classes descritas para o atributo bebida, em reconhecer suas amostras, os 30 compostos voláteis utilizados na discriminação destas classes podem ser apresentados como marcadores importantes deste atributo.

Para o atributo qualidade global, a sensitividade também foi de $100 \%$ para as duas categorias de café (alta e baixa qualidade global).

Tabela 3. Sensitividade e especificidade para os modelos de aroma, bebida e qualidade global

\begin{tabular}{lccccc}
\hline Modelos & Classes & PCs & Distância crítica $\left(S_{\text {crit }}\right)$ & Sensitividade (\%) & Especificidade (\%) \\
\hline Modelo A & Aroma excelente (1) & 5 & 1,44 & 100 & 45 \\
Aroma & Aroma fraco (2) & 2 & 1,51 & 100 & 78 \\
Modelo B & Bebida mole (1) & 4 & 1,48 & 100 & 90 \\
Bebida & Bebida riada /rio (2) & 3 & 1,34 & 100 & 94 \\
Modelo C & Q.G alta (1) & 3 & 1,56 & 100 & 50 \\
Q. G. & Q. G. baixa (2) & 3 & 1,38 & 100 & 90 \\
\hline
\end{tabular}


Contudo, na especificidade a classe de cafés com alta qualidade global aceitou 50\% das amostras de baixa qualidade global, enquanto a classe de cafés de baixa qualidade global apresentou especificidade seletiva reconhecendo todas as amostras de sua classe e apenas $10 \%$ das amostras de alta qualidade global.

\section{CONCLUSÕES}

De acordo com as três análises de componentes principais dos perfis cromatográficos de voláteis de cafés torrados, os compostos: metilpirrol, pirrol, ciclopentanona, di-hidro-2-metil-3-furanona, furfural, 2-etil-5-metilpirazina, 2-etenil-n-metilpirazina, 5-metil-2propionilfurano e mais 5 compostos não identificados podem ser considerados como possíveis marcadores para a diferenciação da qualidade da bebida segundo o seu aroma, qualidade global e sabor. Os compostos pirrol, metilpirrol e o ciclopentanona apresentaram efeito negativo e os demais têm efeito positivo nos 3 atributos.

As figuras de mérito calculadas demonstraram ainda que os 30 compostos voláteis utilizados na discriminação das amostras segundo o atributo bebida são extremamente seletivos para suas classes, ou seja, fortes marcadores de qualidade.

\section{MATERIAL SUMPLEMENTAR}

No material suplementar disponível em http://quimicanova.sbq. org.br, com acesso livre, na forma de arquivo PDF, é apresentado esquematicamente o alinhamento de um vetor desalinhado $\mathbf{x}_{d} \mathrm{em}$ função de um vetor de referência $\mathbf{x}_{r}$, de mesmo comprimento (Figura 1S). Além disso, é apresentado também (Figura 2S) o alinhamento de um vetor desalinhado com número menor de variáveis do que o vetor de referência.

\section{REFERÊNCIAS}

1. Buffo, R; Cardelli-Freire, C.; Flavour Frag. J. 2004, 19, 99.

2. De Maria, C. A. B.; Moreira, R. F. A.; Trugo, L. C.; Quim. Nova 1999, 22, 209.

3. http:// www.abic.com.br acessada em Março 2009.

4. Ribeiro, J. S.; Augusto, F.; Salva, T. J. G.; Thomaziello, R. A.; Ferreira, M. M. C.; Anal. Chim. Acta 2009, 634, 172.

5. Rocha, S.; Maetzu, L.; Barros, A.; Cid, C.; J. Sci. Food Agric. 2004, 84, 43.

6. Agresti, P. D. C. M.; Franca, A. S.; Oliveira, L. S.; Augusti, R.; Food Chem. 2008, 106, 787.

7. Toci, A. T.; Farah, A.; Food Chem. 2008, 108, 1133.

8. Bicchi, C. P.; Ombretta, M. P.; Pellegrino, G. M.; Vanni, A. C.; J. Agric. Food Chem. 1997, 45, 4680.

9. van Nederkassel, A. M.; Daszykowski, M.; Eilers, P. H. C.; Vander Heyden, Y.; J. Chromatogr., A 2006, 1118, 199.
10. Nielsen, N. P. V.; Carstensen, J. M.; Smedsgaard, J.; J. Chromatogr., A 1998, 805, 17.

11. Pravdova, V.; Walczak, B.; Massart, D. L.; Anal. Chim. Acta 2002, 456, 77.

12. Tomasi, G.; van den Berg, F.; Andersson, C.; J. Chemom. 2004, 18, 231.

13. Kassidas, A.; MacGregor, J. F.; Taylor, P. A.; AIChE J. 1998, 44, 864.

14. Eilers, P. H. C.; Anal. Chem. 2004, 76, 404.

15. Forshed, J.; Schuppe-Koistinen, I.; Jacobsson, S. P.; Anal. Chim. Acta 2003, 487, 189 .

16. Walczak, B.; Wu, W.; Chemom. Intell. Lab. Syst. 2005, 77, 173.

17. Daszykowski, M.; Walczak, B.; Trends Anal. Chem. 2006, 25, 1081.

18. Ribeiro, J. S.; Teófilo, R. F.; Augusto, F.; Ferreira, M. M. C.; Chemom. Intell. Lab. Syst. 2010, 102, 45

19. Zambonin, C. G.; Balest, L.; De Benedetto, G. E.; Palmisano, F.; Talanta 2005, 66, 261.

20. Ryan, D.; Shellie, R.; Tranchida, P.; J. Chromatogr., A 2004, 1054, 57.

21. van der Dool, H.; Kratz, P. D.; J. Chromatogr. 1963, 2, 463.

22. Wise, B. M.; Gallagher, N. B.; Bro, R.; Shaver, J. M.; Windig, W.; Koch, R. S.; PLS_Toolbox 3.5, for Use with Matlab ${ }^{T M}$, Eigenvector Research, 2004.

23. Ferreira, M. M. C.; Antunes, A. M.; Melgo, M. S.; Volpe, P. L O.; Quim. Nova 1999, 22, 724.

24. Savitzky, A.; Golay, M. J. E.; Anal. Chem. 1964, 36, 1627.

25. van Nederkassel, A. M.; Xu, C. J.; Lancelin, P.; Sarraf, M.; MacKenzie, D. A.; Walton, N. J.; Bensaid, F.; Lees, M.; Martin, G. J.; Desmurs, J. R.; Massart, D. L.; Smeyers Verbeke, J.; Vander Heyden, Y.; J. Chromatogr. A 2006, 1120, 291.

26. Meléndez, M.; Ortíz, M. C.; Sánchez, M.; Sarabia, L.; Iñiguez, M.; Quim. Anal. 1999, 18, 119.

27. Coomans, D.; Broeckaert, I.; Potential pattern recognition in chemical and medical decision making, $1^{\text {st }}$ ed., John Wiley: Letchworth, 1986.

28. Adams, R. P.; Identification of essential oil components by gas chromatography/ mass spectroscopy, $4^{\text {th }}$ ed., Allured Publishing Corporation: Carol Stream, 1995.

29. http://webbook.nist.gov/chemistry/ acessada em Março 2010.

30. Semmelroch, P.; Grosch, W.; J. Agric. Food Chem. 1996, 44, 537.

31. Czerny, M.; Grosch, W.; J. Agric. Food Chem. 2000, 48, 868.

32. Morais, S. A. L.; Aquino, F. J. T.; Chang, R.; Nascimento, E. A.; Oliveira, G. S.; Santos, N. C.; Coffee Sci. 2007, 2, 97.

33. Flament, I.; Coffee Flavor Chemistry, $1^{\text {st }}$ ed., British Library Cataloguing: London, 2002.

34. Lopez-Galilea, I.; Fournier, N.; Cid, C.; Guichard, E.; J. Agric. Food Chem. 2006, 54, 8560.

35. Franca, A. S.; Oliveira, L. S.; Oliveira, R. C. S.; Agresti, P. C. M.; Augusti, R.; J. Food Eng. 2009, 92, 345.

36. Oliveira, R. C. S.; Oliveira, L. S.; Franca, A. S.; Augusti, R.; J. Food Comp. Anal. 2009, 22, 257. 


\section{USO DE PERFIS CROMATOGRÁFICOS DE VOLÁTEIS DE CAFÉS ARÁBICAS TORRADOS PARA A DIFERENCIAÇÃO DAS AMOSTRAS SEGUNDO O SABOR, O AROMA E A QUALIDADE GLOBAL DA BEBIDA}

Juliano S. Ribeiro, Fabio Augusto e Márcia M. C. Ferreira*

Instituto de Química, Universidade Estadual de Campinas, CP 6154, 13083-970 Campinas - SP, Brasil

Terezinha J. G. Salva

Centro de Análise e Pesquisa Tecnológica do Agronegócio do Café "Alcides Carvalho", Instituto Agronômico de Campinas, CP 28, 13001-970 Campinas - SP, Brasil.

A Figura 1S apresenta esquematicamente o alinhamento de um vetor desalinhado $\mathbf{x}_{d}$ (linha cinza escura contínua) em função de um vetor de referência $\mathbf{x}_{r}$ (linha preta contínua), de mesmo comprimento. Utilizando $N=1$ e $s=2$, observam-se os possíveis deslocamentos indicados em linhas pontilhadas, e o melhor alinhamento $M-s$ (linha cinza clara pontilhada sobreposta à linha preta contínua) no quadro $\mathbf{B}$ da Figura $2 \mathrm{~S}$.
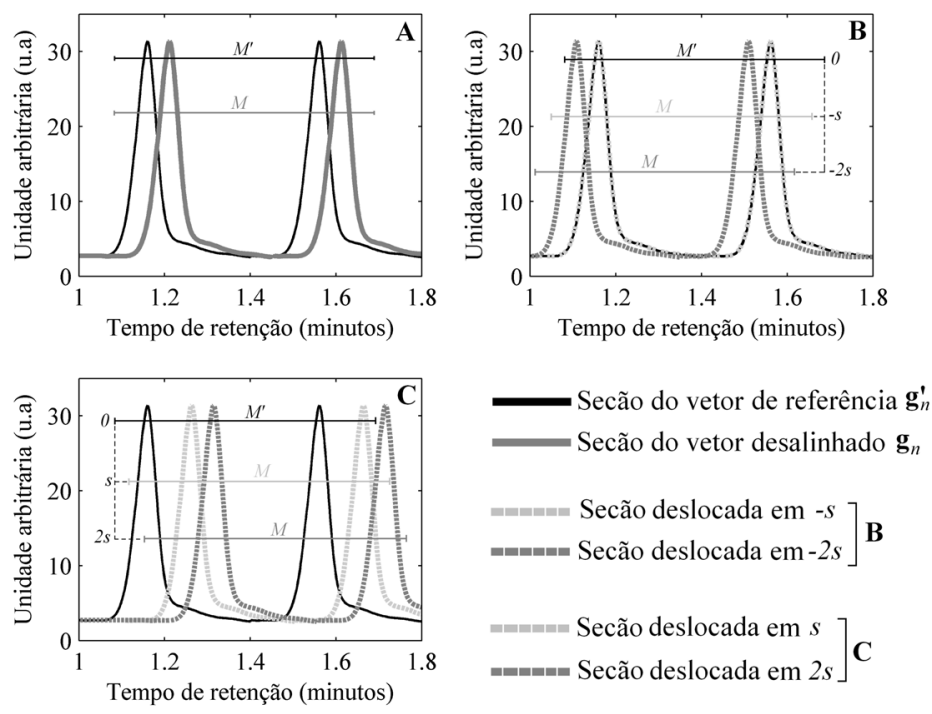

Figura 1S. Representação esquemática de uma região expandida de 2 cromatogramas, um de referência e outro desalinhado durante o processo de alinhamento utilizando-se o método COW; $\mathbf{m}$ e $\mathbf{m}$ ' são os comprimentos de seção de $\mathbf{x}_{d}$ e $\mathbf{x}_{r}$, respectivamente, e s o parâmetro de deslocamento

A Figura 2S apresenta esquematicamente o alinhamento de um vetor desalinhado com menor número de variáveis do que o vetor de referência.

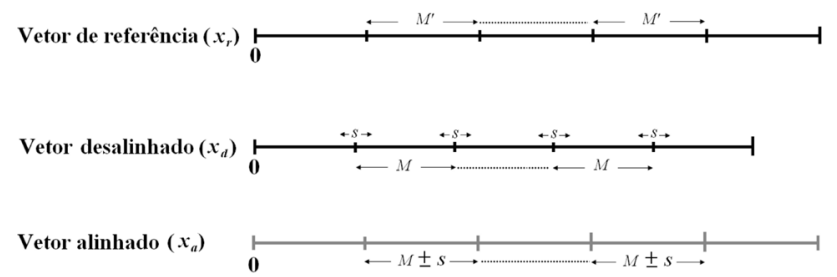

Figura 2S. Representação esquemática do funcionamento do algoritmo COW alinhando sinais de comprimentos diferentes (J diferente de J'e, portanto, M diferente de M') 